\title{
The Aging of the Middle Ear in 129S6/SvEvTac and CBA/CaJ Mice: Measurements of Umbo Velocity, Hearing Function, and the Incidence of Pathology
}

\author{
John J. Rosowski, ${ }^{1,2}$ Kelly M. Brinsko, ${ }^{1}$ Bruce I. Tempel, ${ }^{3,4}$ and Sharon G. Kujawa ${ }^{1,2,5}$ \\ ${ }^{1}$ Eaton-Peabody Laboratory, Massachusetts Eye and Ear Infirmary, Boston, MA 02114, USA \\ ${ }^{2}$ Department of Otology and Laryngology, Harvard Medical School, Boston, MA 02114, USA \\ ${ }^{3}$ Virginia Merrill Bloedel Hearing Research Center, University of Washington, Seattle, WA 98195, USA \\ ${ }^{4}$ Department of Otolaryngology/Head and Neck Surgery, University of Washington School of Medicine, Seattle, \\ WA 98195, USA \\ ${ }^{5}$ Department of Audiology, Massachusetts Eye and Ear Infirmary, Boston, MA 02114, USA
}

Received: 12 September 2002; Accepted: 16 January 2003; Online publication: 14 March 2003

\section{ABSTRACT}

Measurements of umbo velocity and auditory brainstem response (ABR) were made on two different strains of mice, 129S6/SvEvTac (129S6) and CBA/ CaJ $(\mathrm{CB})$, within three different age ranges. The velocity measurements were made with a laser Doppler vibrometer using a semiclosed sound delivery system; the frequency range of accurate velocity measurements is from 1 to $21 \mathrm{kHz}$. The visual detection threshold of the ABR was determined at selected frequencies between 2 and $32 \mathrm{kHz}$. The velocity results suggest a small but significant change in umbo velocity with age in both strains, between the youngest (1.5-3 months) and mid-aged (12-14 months) groups. There is also a clear difference in the umbo velocity in the youngest animals of the two strains, with the 129S6 having more sensitive middle-ear function than the CB. These results support the existence of a small age-related loss in middle-ear sensitivity in the mouse that was first described in the BALB/6J strain. The age-related changes in middleear function observed in both $\mathrm{CB}$ and 129S6 are much smaller than the age-related decreases in ABR. Our results also describe a statistically significant in-

Correspondence to: John J. Rosowski • Eaton-Peabody Laboratory • Massachusetts Eye and Ear Infirmary • 243 Charles Street • Boston, MA 02114. Telephone: (617) 573-4237; fax: (617) 720-4408; email: jjr@epl.meei.harvard.edu crease in the incidence of middle-ear pathology with age in the 129S6.

Keywords: middle ear, aging, presbycusis, middleear infection

\section{INTRODUCTION}

The use of molecular and genetic techniques in investigations of auditory function has led to a huge increase in the number of experimental studies that make use of various strains of mice. These studies include investigations of the development of the ear (Huangfu and Saunders 1983; Doan et al. 1994), the sensitivity of the ear to acoustic trauma ( $\mathrm{Li}$ and Borg 1993; Yoshida et al. 2000; Willott et al. 2000), and the aging of the ear ( $\mathrm{Li}$ and Borg 1991; Doan et al. 1996a). All of these studies depend on the definition of normal function, and it has been repeatedly demonstrated that the ears of different strains of mice show different patterns of development and aging (Jimenez et al. 1999; Zheng et al. 1999).

The CBA/CaJ (CB) strain has been used extensively as the standard for normal hearing in studies of hearing function and how it changes with age ( $\mathrm{Li}$ and Borg 1991; Willott et al. 1992; Jimenez et al. 1999). While 129-derived strains, e.g., 129S6/SvEvTac (129S6), were little used in earlier studies of mouse 
audition, they are becoming quite common in auditory studies (e.g., Zheng et al. 1999; Yoshida et al. 2000) and are prominent in the generation of "knockout" mice for auditory and nonauditory applications. Since it has been demonstrated previously that there are strain-dependent differences in the function of the developing and aging auditory system of different mice ( $\mathrm{Li}$ and Borg 1991; Jimenez et al. 1999), it is important to understand the bases for these differences.

The results in this report are part of a larger study of how differences in the genetic programming within the inner ear affect susceptibility to hearing loss with age and noise exposure. The larger study includes measurements of cochlear and peripheral auditory function, including distortion-product otoacoustic emissions (DPOAE) and auditory brainstem responses (ABR), in aging mice of different genetic backgrounds (Kujawa et al. 2001, 2002). In performing preliminary studies, three questions arose: (1) Are there any age-related alterations in middle-ear function in the different mouse strains? (2) Is there any correlation between age-related changes in the middle ear and other measures of auditory function? (3) Are there age- and strain-related differences in the susceptibility to middle-ear disease?

The first question has been addressed in one mouse strain by Doan et al. (1996a,b), who used measurements of umbo velocity to determine age-related changes in middle-ear sound transmission and found a small $(6-10 \mathrm{~dB})$ reduction in velocity in old $\mathrm{BALB} / \mathrm{c}$ mice in response to tones of $4-12 \mathrm{kHz}$. This result suggests that a $6-10 \mathrm{~dB}$ reduction in auditory sensitivity in aging mice is related to a loss in middleear sound transfer. We investigate this question in two other mouse strains also using measurements of the sound-induced velocity near the center of the tympanic membrane (close to the umbo). This velocity, which is at the input to the ossicular system, is a measure of the transmission of sound between the external ear and the inner ear.

The second question is addressed by investigating the degree of correlation between the age-related changes in umbo velocity we observed with changes in the sound-evoked auditory brainstem response (ABR), a measure of the sensitivity of the entire auditory periphery to sound. Correlations between the age-dependent ABR and umbo velocity have been shown in some developing animals (Relkin and Saunders 1981; Doan et al. 1994, 1996b).

With regard to the third question, McGinn et al. (1992) noted an increased incidence of otitis media in $\mathrm{CBA} / \mathrm{J}$ with little incidence in $\mathrm{CBA} / \mathrm{CaJ}$ (the $\mathrm{CB}$ of our study), and our own observations suggest that the 129S6 is more susceptible to middle-ear disorders than CB, especially in older mice. The purpose of this

\section{TABLE 1}

Number of normal ears for ABR testing

\begin{tabular}{cccc}
\hline & Young & Middle age & Old age \\
\hline 129S6 & 55 & 45 & 14 \\
CB & 83 & 20 & 16 \\
\hline
\end{tabular}

study is to investigate these questions regarding agerelated changes in middle-ear function and the susceptibility to middle-ear disease in the $\mathrm{CB}$ and the 12956 strains.

\section{METHODS}

The two strains of mice (CB and 129S6) were investigated in three different age groups: (1) 1.5-3 months, (2) 12-14 months, and (3) 18-24 months. Mice reach sexual maturity within 3.5-4 weeks after birth and can live for 2.5-3.5 years in captivity; therefore, our three groups represent young adults, middle-aged, and old mice. All mice were born and reared in an AAALAC-approved Specific Pathogen Free (SPF) facility at the University of Washington from stock originally obtained from Jackson Labs (CB) and Taconic (129S6). All breeding and husbandry procedures were approved by the Animal Care and Use Committee of the University of Washington. The mice from the two strains were housed, fed, and otherwise treated identically and were airfreighted to the Massachusetts Eye and Ear Infirmary several days to two weeks prior to electrophysiologic and mechanoacoustic measurements. Upon arrival at the Massachusetts Eye and Ear Infirmary Animal Facility, the mice were kept in a dedicated "isolation" room until the time of the measurements.

Data from two subsets of the bred and transported mice were used in our measurements. A larger subset with normal middle ears that included from 14 to 83 mice in each of the three age groups and two strains was used in ABR measurements (Table 1). A smaller subset was examined for middle-ear measurements. The middle-ear subset was filled by a sequential sampling of the mice that arrived at the Infirmary from November 2001 through May 2002. The normal ears within the middle-ear subset are also included in the ABR subset.

\section{Auditory brainstem response measurements}

All animals were prepared and tested using techniques described previously (Parkinson et al. 2001). Mice were anesthetized (ketamine, $100 \mathrm{mg} / \mathrm{kg}$ IP; xylazine, $10 \mathrm{mg} / \mathrm{kg}$ IP with booster injections $1 / 3$ to $1 / 2$ the original dose as needed) and a small slit was 
made in the external canal to accommodate microscopic examination of the tympanic membrane (TM) and accurate placement of the sound delivery system. Only animals with normal-appearing TMs are included in the mean measurements. ABRs were elicited using 5-ms tone pips (4-45 kHz; 0.5-ms rise/fall; $\cos ^{2}$ shaping; with alternating polarity), delivered at a rate of $40 / \mathrm{s}$. Responses were detected by subdermal needle electrodes (vertex to ventrolateral to the test ear with base-of-tail ground), amplified $(10,000$ times), filtered (0.1-3-kHz bandpass), and averaged (512 sweeps at each frequency-level combination, repeated as needed for levels bracketing threshold; artifact reject $=15 \mu \mathrm{V}$ peak-to-peak) with $\mathrm{A}-\mathrm{D} / \mathrm{D}-\mathrm{A}$ boards (National Instruments, Austin, TX; 16-bit) in a LabVIEW-driven data acquisition system. ABR "thresholds," defined as the lowest sound level at which response peaks were clearly present, were read by eye from stacked waveforms obtained in 5-dB steps at sound pressures from about $10 \mathrm{~dB}$ below threshold to $80 \mathrm{~dB}$ SPL. Repeated threshold determinations showed $\pm 5 \mathrm{~dB}$ repeatability. Most of these mice were allowed to recover from anesthesia and returned to the colony; however, a temporally contiguous subset was prepared for middle-ear measurements, including mice that had failed the initial screen for middleear pathology. All surgical and measurement procedures were approved by the Animal Care and Use Committee of the Massachusetts Eye and Ear Infirmary.

\section{Surgical preparation for the middle-ear} measurements and the assessment of TM health

The middle-ear measurements were a terminal procedure. Each mouse was anesthetized as previously described. After induction of anesthesia, the pinna and cartilaginous external ear canal were removed to expose the pars tensa and pars flaccida of the tympanic membrane (TM).

Stereo-microscopic observations were then made of the health of the TM and middle ear by noting the color and appearance of the two TM segments, the existence of any fluid in the middle ear cavity (usually observable via the presence of a meniscus on the $\mathrm{TM}$ ), and the presence (or absence) and degree of retraction of the pars flaccida. A "normal" TM was transparent or slightly translucent, with no visible blood vessels on the membrane itself (the manubrium of the malleus may appear vascular) and no fluid visible in the middle-ear space. Because anesthesia inhibits the normal eustachian tube function, retraction of the pars flaccida was observed in many ears; however, that retraction was relieved when the normal middle-ear air space was vented. Pathologic TMs ranged in color from cloudy to opaque white or yel- low. A pathologic TM might also appear thickened or edematous and often had blood vessels visible throughout the surface of the TM. A fluid meniscus was sometimes visible through the TM, though ears that were filled with fluid showed no meniscus.

After exposing the TM, the posterior-inferior bullar wall was exposed and a small hole (about 0.55 $\mathrm{mm}$ in diameter) was made using a sharp scalpel blade. The middle-ear cavity was examined for fluid or other pathologic matter (visually and by probing with fine paper points), and, if found to be clear, a polyethylene vent tube (i.d. $=0.28 \mathrm{~mm}$, o.d. $=0.61$ $\mathrm{mm}, 64 \mathrm{~mm}$ long) was press-fitted into the bullar hole to act as static pressure relief while closing the bulla at acoustic frequencies (Lynch et al. 1994). Only ears with air-filled middle ears and no TM pathology were included in the normal groups.

\section{Measurements of umbo velocity}

A laser Doppler vibrometer (LDV) (Polytec PI Inc., Tustin, CA; OFV 501 fiber interferometer with an OFV 2600 controller) was used to measure the soundinduced velocity near the center of the tympanic membrane. A small piece of autoreflective tape (usually containing three to four 50- $\mu$ m-diameter balls) was placed on the manubrium, as close to the umbo as possible. (In mice the umbo is close to the center of the TM.) A cylindrical brass coupler with a side-arm sound entry port was used both to steady the head of the mouse and to control the sound field around the entrance to the ear canal (Fig. 1). A broad band $(0.050-25 \mathrm{kHz})$ chirp stimulus was generated by a DOS-based desktop computer with a DSP16+ signal processing board (Ariel Corporation, Cranbury, NJ) using SYSid (SYSid Labs, Richmond, CA). The output signal from the DSP16+ was amplified by a power amplifier and coupled to a Beyerdynamic earphone (Farmingdale, NY; DT-48) that generated the acoustic signal presented to the ear. The LDV was focused onto the umbo reflector through the open back of the brass coupler and the response was measured by SYSid. After the laser measurement, the sound pressure within the ear canal was measured by placing a probetube microphone into the entrance of the canal and performing a separate measurement. Noise-floor measurements, taken when the stimulus was off, were also obtained to determine the range in which the LDV and probe microphone measurements would not be compromised by noise interference. The combination of small velocities and low-frequency noise on the laser channel limited our ability to accurately measure umbo velocity at frequencies below $1 \mathrm{kHz}$. [At $1 \mathrm{kHz}$, the signal-to-noise ratio of the laser measurements was about $3(10 \mathrm{~dB})$. At lower frequencies the ratio was smaller. At higher frequencies 


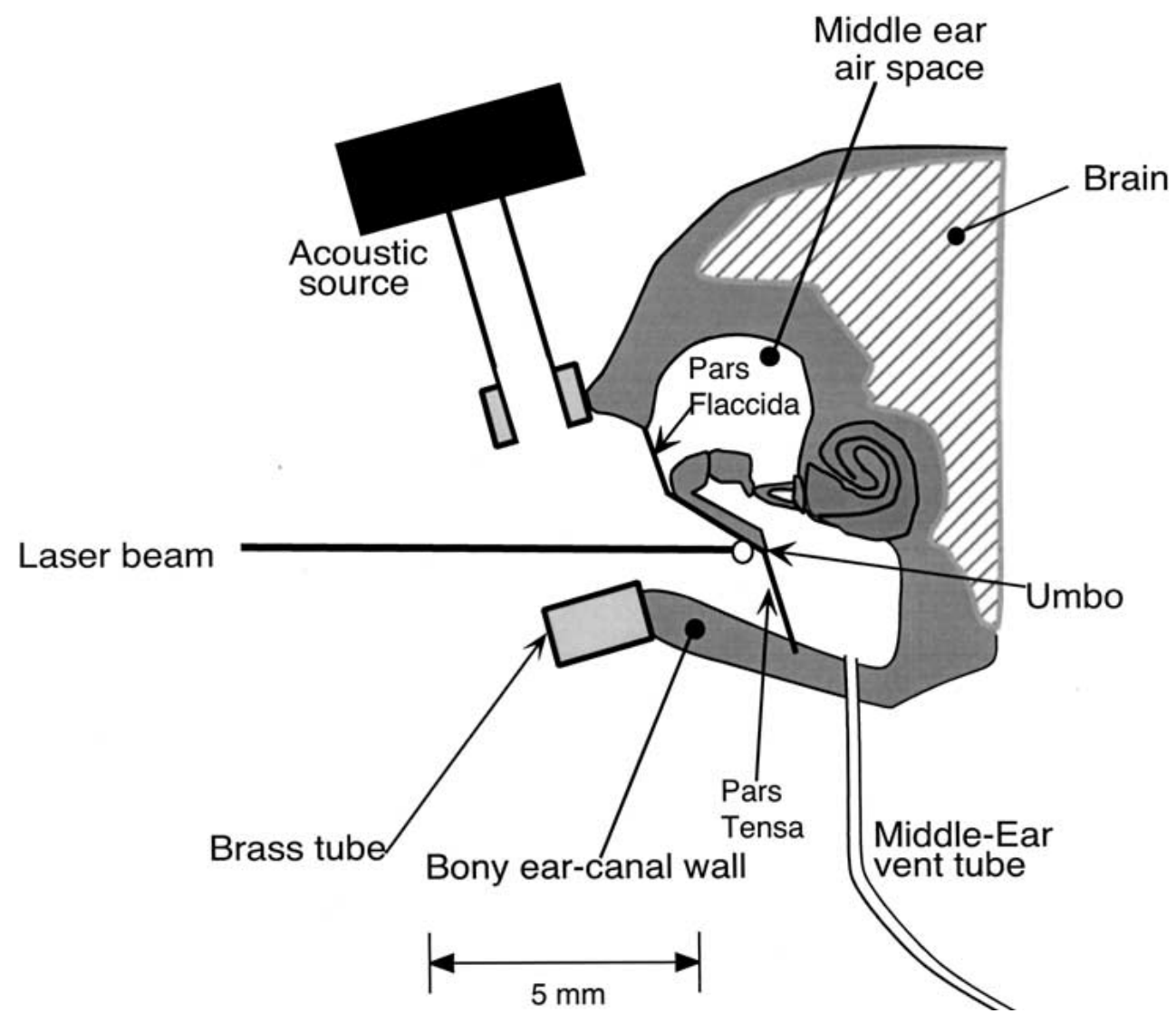

FIG. 1. Methods: The cartilaginous ear canal is removed and the middle-ear air space is vented with a long thin tube. An open-backed brasstube coupler with a side-arm sound source is sealed over the entrance of the bony ear canal. Reflective balls are placed on the latter surface of the TM near the umbo. The laser is focused on the TM reflector through the open back of the brass-tube coupler.

TABLE 2

\begin{tabular}{|c|c|c|c|c|c|c|}
\hline \multicolumn{7}{|c|}{ Number of ears examined for middle-ear testing } \\
\hline & \multicolumn{2}{|c|}{ Young } & \multicolumn{2}{|c|}{ Middle age } & \multicolumn{2}{|c|}{ Old age } \\
\hline & Total & Normal & Total & Normal & Total & Normal \\
\hline $129 S 6$ & 14 & 11 & 27 & 20 & 22 & 7 \\
\hline CB & 11 & 11 & 10 & 8 & 12 & 10 \\
\hline
\end{tabular}

the ratio was larger.] The combination of poor earphone output and microphone noise limited our ability to accurately determine ear canal sound pressure at frequencies above $21 \mathrm{kHz}$. Umbo velocity transfer functions (with magnitude and phase angle) for the 1-21-kHz range were computed from the ratio of the complex umbo velocity and ear-canal sound pressure at each measured frequency.

In testing for differences across strain and age, only animals with healthy ears, free of pathology, were included. Table 2 lists the total number of ears prepared for middle-ear studies and the number of normal ears found in each strain and age group. Table 3 lists the mean, range, and standard deviation of the ages of all of the ears examined in the six middle-ear study groups; there are small, insignificant differences between the age statistics for the total number of examined ears and that subset of normal ears within each group. The mean and 95\% confidence interval of the mean of the magnitudes and angles of the normal-ear umbo velocity transfer functions, at each frequency, were computed for each of the six groups. A two-way analysis of variance (ANOVA) accounting for both age and strain differences was performed on the transfer function magnitudes at four different frequencies for determination of the significance of variation.

Laser Doppler vibrometry measurements were also made on some of the animals with demonstrated pathology of the tympanic membranes and middle ear cavities so that we could assess the effects of such pathologies on the umbo velocity transfer function. 
TABLE 3

\begin{tabular}{cccc}
\hline \multicolumn{4}{c}{ TABLE 3 } \\
\hline Distribution of ages within the middle-ear testing group \\
Young & Middle age & Old age \\
& Range & Range & Range \\
Mean $\pm S D$ & Mean $\pm S D$ & Mean $\pm S D$ \\
\hline $129 S 6$ & $1.5-3$ months & $12-14$ months & $18-24$ months \\
& $1.6 \pm 0.23$ & $12.4 \pm 0.72$ & $21.7 \pm 2.88$ \\
& $1.5-3$ months & $12-14$ months & $18-20$ months \\
& $2.0 \pm 0.76$ & $13.2 \pm 0.78$ & $18.6 \pm 0.57$ \\
\hline
\end{tabular}

While compiling these data, we confirmed our earlier impression that middle-ear pathology occurred more frequently in the 129S6 strain, especially in older ears. A series of $\Gamma^{2}$ tests were performed on the incidence of normal and pathologic ears to determine the significance of the observed differences in the distribution of ear disease in our six groups.

\section{RESULTS}

We measured $A B R$ and sound-induced velocity of the umbo in 129S6 and CB mice at three ages. The ABR results, discussed later, demonstrate a significant agerelated hearing loss in both strains that is larger in the 129S6. The middle-ear results suggest that there are small but significant age-dependent differences in middle-ear function within each strain and that there are also differences between the strains. The strainrelated differences in the sensitivity of the middle ear and the ABR are not correlated, and the age-related losses in ABR sensitivity are much greater than the age-related decreases in middle-ear velocity that we observed. Significant differences in the incidence of middle-ear pathology were also found with age and strain.

\section{Differences in middle-ear function with age} within 12956

An issue in any comparison of functional variations with strain and age is the variation within each subset of the population. Figure 2 shows the individual responses as well as the mean and $95 \%$ confidence interval of the mean of the magnitude and angle of the umbo velocity transfer function determined in 11 normal 129S6 mice between 6 and 8 weeks of age postpartum. At frequencies below $8 \mathrm{kHz}$, the transfer functions are consistent with a stiffness-controlled tympanic membrane where the magnitude is proportional to frequency (a log-log slope of 1 ) and the angle is near 0.25 period. At higher frequencies, the growth of velocity magnitude with frequency slows

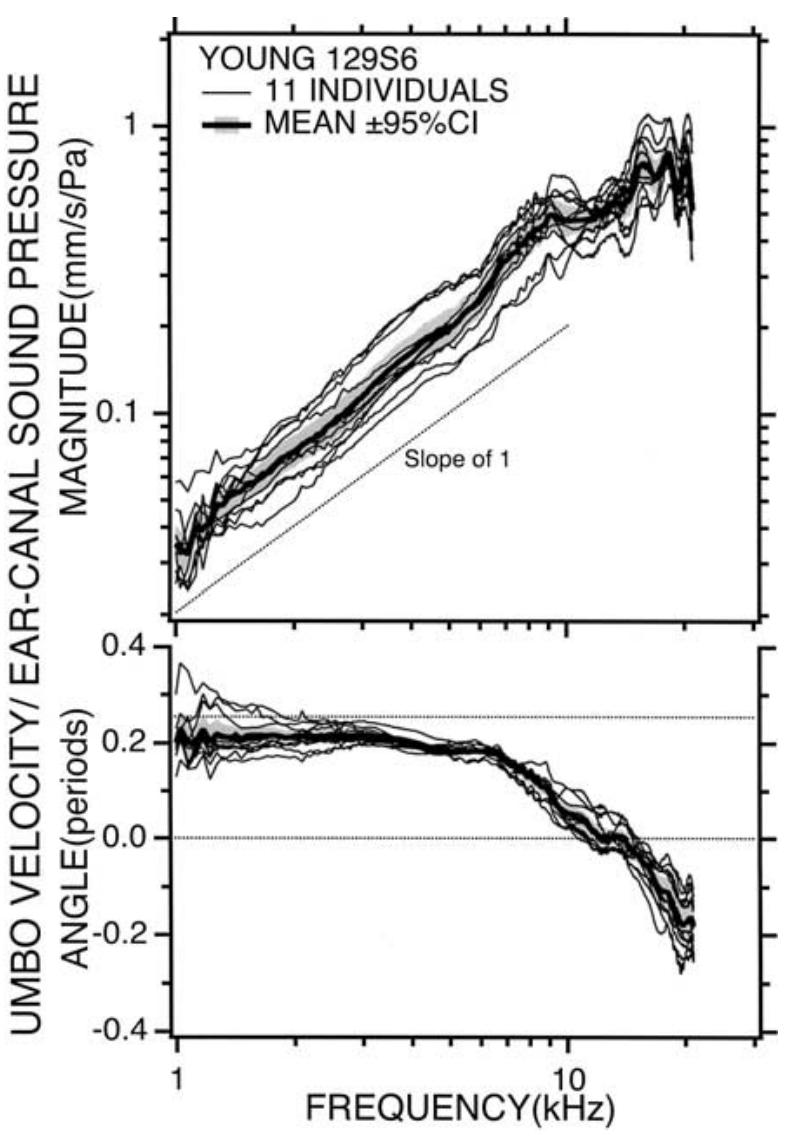

FIG. 2. Umbo velocity transfer function in 11 young 12956 . The transfer function is the complex ratio of the measured umbo velocity and the sound pressure near the tympanic membrane. The magnitude is the ratio of the magnitudes of the velocity and pressure. The angle is the phase difference between the measured velocity and sound pressure. The dashed line in the magnitude panel illustrates a log-log slope of 1 that is indicative of a magnitude that grows proportionately with frequency. The dashed lines in the angle panel are at values of 0.25 (the angle associated with compliant behavior) and 0 periods. The thick dark line shows the computed mean from the 11 ears. The shaded region shows the 95 confidence interval around the mean.

and the angle decreases past -0.20 with an angle of zero at about $12 \mathrm{kHz}$. The magnitude at $12 \mathrm{kHz}$ has a value of about $0.5 \mathrm{~mm} \mathrm{~s}^{-1} \mathrm{~Pa}^{-1}$. The range of magnitudes in the 11 ears covers about a factor of $2(6 \mathrm{~dB})$, 


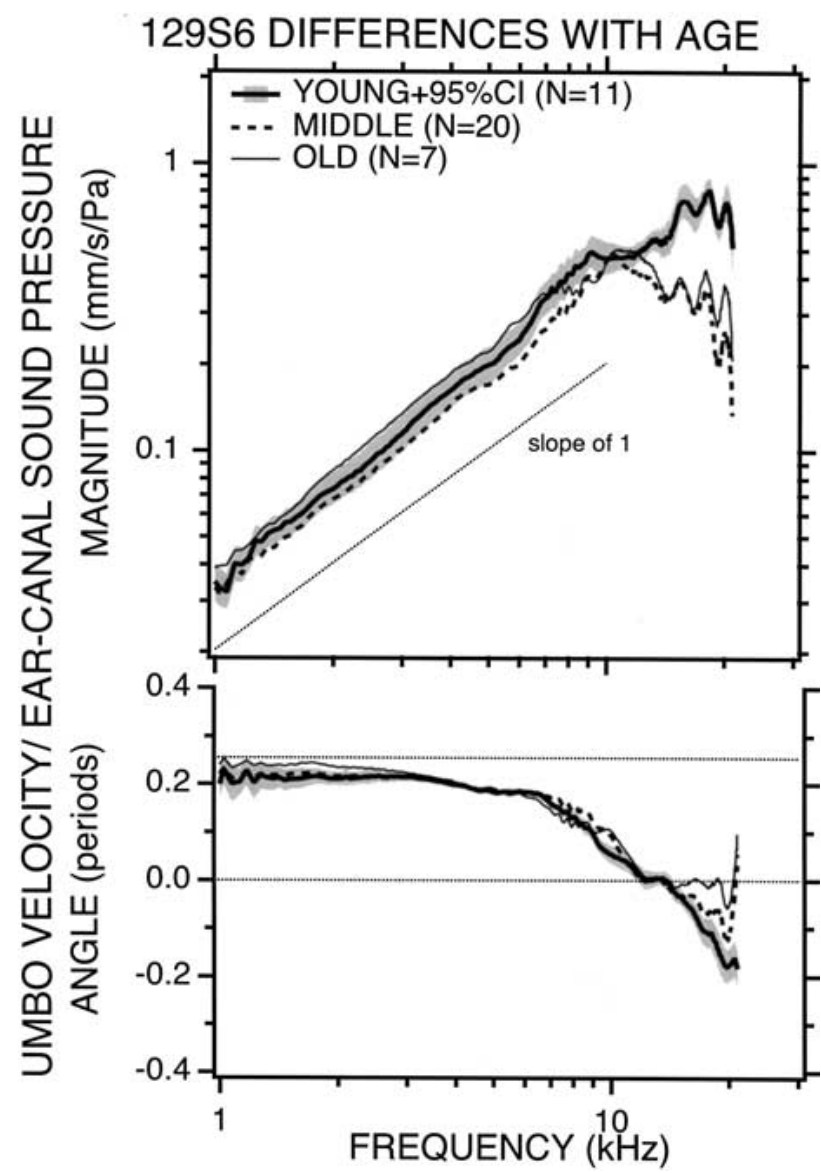

FIG. 3. Comparisons of the mean umbo velocity transfer function in the three age groups of 129S6. Panels for magnitude and angle as in Figure 2. The three lines show the mean velocity magnitude and angle of the three age groups, while the shaded regions shows the 95 confidence interval around the young group's mean. The dashed line in the magnitude panel illustrates a log-log slope of 1 that is indicative of a magnitude that grows proportionately with frequency. The dashed lines in the angle panel are at values of 0.25 (the angle associated with compliant behavior) and 0 periods.

while the range of the angles covers about 0.06 period $\left(\sim 25^{\circ}\right)$.

The means and normalized standard deviations of the three age groups at four selected frequencies are noted in Table 4 and the means of the three age groups of 129S6s are illustrated in Figure 3. While the data suggest a great similarity in the umbo velocity in the three ages, the velocity magnitudes measured at frequencies above $12 \mathrm{kHz}$ are significantly smaller in the two older groups. The mean magnitude of the umbo velocity of the middle group is generally the smallest at each frequency (though these values are not, in general, statistically different from the old-age group). The angles of the transfer functions show significant but less regular differences between the three ages. The youngest group has the lowest mean angles at most frequencies. The frequency where the angle first reaches 0 (near $12 \mathrm{kHz}$ ) is similar in all 


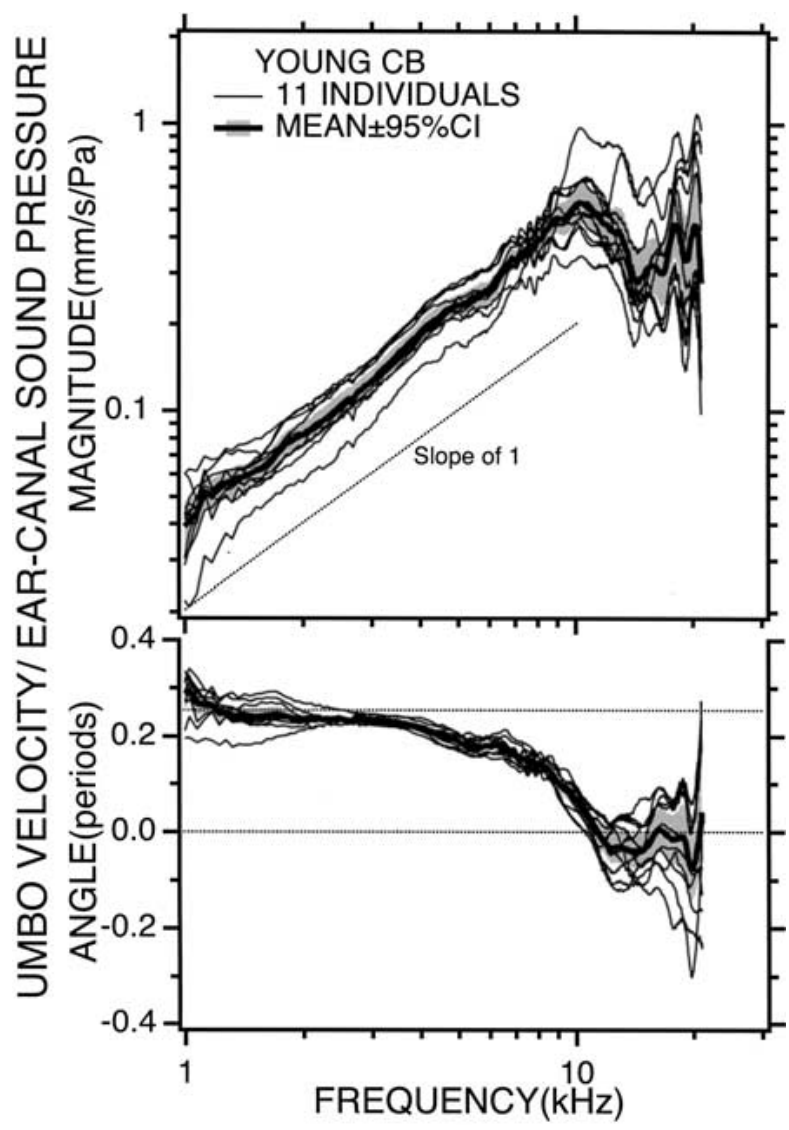

FIG. 4. Umbo velocity transfer function in 11 young CB plotted as in Figure 2.

three age groups. The angles at the highest frequencies are closer to 0 in the old group. Table 4 points out that the standard deviation of the magnitudes normalized by the mean in the three 129S6 age groups does not change regularly with frequency, but that the normalized deviation is smaller in the youngage group. There is, however, a frequency dependence of the deviation in angle, where the deviation normalized by the mean is smallest at $4 \mathrm{kHz}$ and increases with frequency. (Large normalized deviations in angle occur when the mean angle is near 0.)

Differences in middle-ear function with age within CB

An example of the variation observed within the young group of $\mathrm{CB}$ mice is shown in Figure 4. The umbo velocity transfer function in the 11 young $\mathrm{CB}$ mice again appears stiffness-controlled at frequencies below $8 \mathrm{kHz}$, though there is some inconsistent deviation from a unit magnitude slope and angle near 0.25 period below $2 \mathrm{kHz}$ that may be attributed to the decreased signal to noise in the velocity measurements at these low frequencies. The range of magnitudes at any frequency below $8 \mathrm{kHz}$ is about a factor

\section{CB DIFFERENCES WITH AGE}

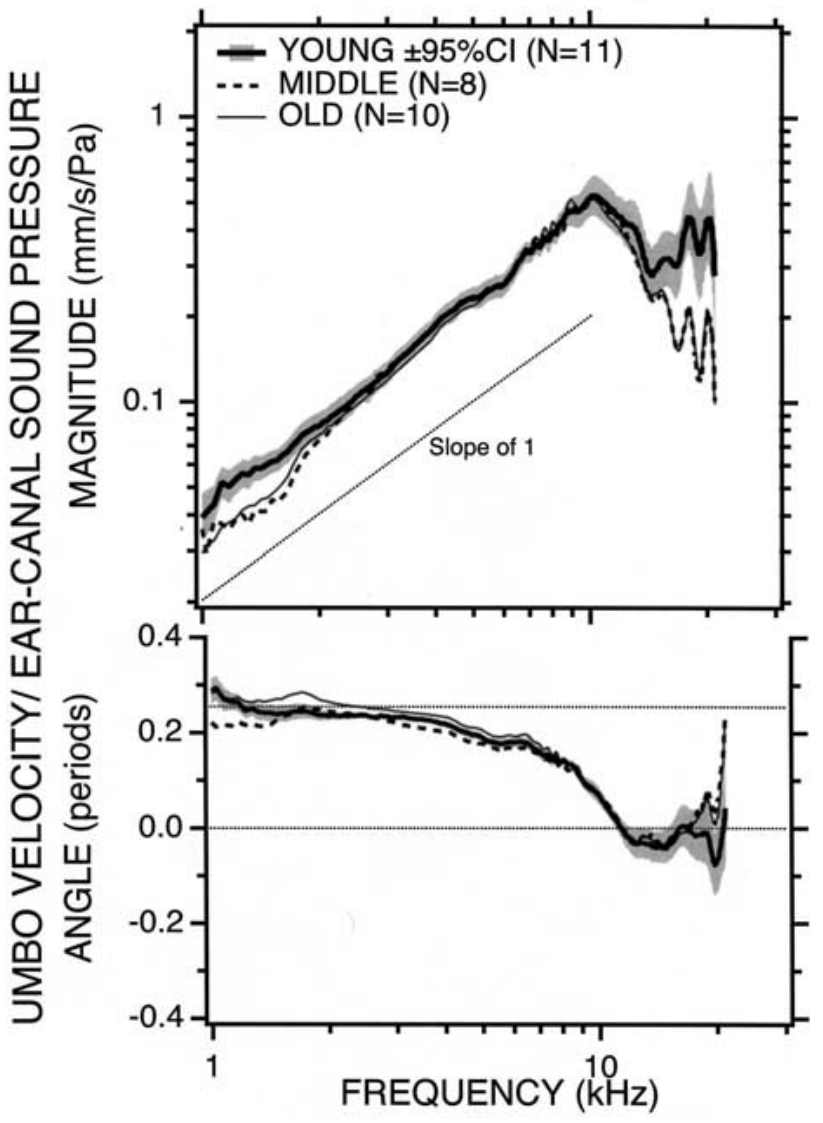

FIG. 5. Comparisons of the mean umbo velocity transfer function in the three age groups of CB plotted as in Figure 3.

of 2 (as in the young 129S6) and the range of angles is again about 0.06 period. At frequencies above 8 $\mathrm{kHz}$, there is an increase in the variability in these measurements, with the range in magnitude expanding to about a factor of 4 and the range in angle growing to about 0.25 period at the highest frequencies. The frequency where the mean angle is 0 is about $12 \mathrm{kHz}$ and the mean magnitude at this frequency is about $0.5 \mathrm{~mm} \mathrm{~s}^{-1} \mathrm{~Pa}^{-1}$.

Age-related differences in the umbo velocity transfer function in CB are illustrated in Figure 5 and quantified in the right-hand side of Table 4. While the velocities in the three age groups are generally similar in magnitude and angle, especially at frequencies less than $15 \mathrm{kHz}$, there are significant differences in velocity magnitude at frequencies above $15 \mathrm{kHz}$ between the youngest and the two older groups. Like the 129S6 data, the youngest CB have the largest magnitudes at frequencies above $15 \mathrm{kHz}$ and these magnitudes are significantly larger than those of either of the other ages. The differences between the middle- and old-age groups at any frequency are not significant. Differences between the angles of the umbo velocity transfer function in the 
TABLE 5

The effects of age: two-way ANOVA results

\begin{tabular}{ccccc}
\hline & \multicolumn{3}{c}{ Frequency $(\mathrm{Hz})$} \\
\cline { 2 - 5 } & 4000 & 8000 & 12000 & 16000 \\
\hline & & Magnitude & $p<0.0001$ \\
Age & NS & NS & NS & $p<0.0001$ \\
Strain & NS & NS & NS & NS \\
Angle & $N S$ & NS & NS & NS \\
Age & N & NS & $p<0.02$ & \\
\hline
\end{tabular}

three age groups are generally small and not significant with no clear trend with age. Table 4 points out that standard deviations of the magnitudes normalized by the mean in the three $\mathrm{CB}$ age groups change regularly with frequency, but not with age, with the smallest normalized deviations at $4 \mathrm{kHz}$ and the largest normalized deviations at $16 \mathrm{kHz}$. There is again a frequency dependence of the variation in angle, where the deviation relative to mean is smallest at $4 \mathrm{kHz}$ and increases with frequency.

\section{Differences in middle-ear function between the strains}

Careful comparisons of Figures 2-5 show strain-associated differences in umbo velocity at the different ages. The mean magnitude of the umbo velocity at frequencies above $12 \mathrm{kHz}$ of the youngest group of the CB strain is significantly smaller than that of the young 129S6 (Fig. 2 and 4). Smaller but still significant differences are also visible in the middle- and old-age groups of the two strains (Figs. 3 and 5). Another statistical difference between the two strains is visible in Table 4 . The normalized deviations in magnitude observed at 4 and $8 \mathrm{kHz}$ in the $\mathrm{CB}$ mice are smaller than the normalized deviations in the 129S6 mice at the same frequencies, while at 12 and $16 \mathrm{kHz}$, the range of the normal deviations overlaps in the two species. This distribution of variance suggests that low-frequency middle-ear function is more variable in the 129S6 strain, but that the variance at frequencies above $10 \mathrm{kHz}$ is similar in the two strains.

These observations were further tested by a twoway (age group and strain) analysis of variance of the magnitude and angle data from all normal ears at four selected frequencies $(4,8,12$, and $16 \mathrm{kHz})$. The results of this analysis are summarized in Table 5 . A significant variation $(p<0.05)$ in magnitude associated with differences between the two strains was found only at $16 \mathrm{kHz}$. Significant variations in angle at 4 and $12 \mathrm{kHz}$ were also associated with differences in strain. Differences in age were associated only with significant magnitude variations at $16 \mathrm{kHz}$, consistent with the high-frequency age-related differences observed in Figures 3 and 5. No significant interactions between age and strain were observed.

Differences in ABR threshold with age and strain: comparisons to differences in umbo velocity

We also measured ABR thresholds in a superset of animals that contain all of the normal ears of our three age groups of 129S6 and CB mice. These measurements are part of a more complete study in preparation, which compares $\mathrm{ABR}$ and distortion product otoacoustic emissions in mice of different strains, ages, and genetic backgrounds. Those studies (Kujawa et al. 2001, 2002) show that young CB have somewhat lower ABR thresholds than young 129S6 at frequencies below $20 \mathrm{kHz}$ and that, at higher frequencies, 129S6 thresholds increase more than the CB. At older ages, CB animals show relatively small threshold elevations of 5-20 dB. These shifts involve the entire frequency range of tests. The 129S6 demonstrate larger age-related shifts than the CB. In this report we compare the differences in the measured ABR thresholds for similar groupings of animals (Fig. $6)$.

One of the larger differences in middle-ear function that we have observed is the difference in the magnitude of umbo velocity between young 12956 mice and young $\mathrm{CB}$ mice. The ratio of the velocities of the young 129S6 and young $\mathrm{CB}$ groups (in $\mathrm{dB}$ ) is compared to the difference in ABR threshold between these two age and strain groups in Figure 6A. The $\mathrm{dB}$ difference in sensitivity is defined such that a higher threshold (decreased sensitivity) for the 129S6 compared to the $\mathrm{CB}$ results in a negative $\mathrm{dB}$ value. In the young CB and 129S6, ABR thresholds are significantly higher in the 129S6 (leading to a decreased sensitivity) at all frequencies tested. The thresholds of the $129 \mathrm{~S} 6$ group are $6-8 \mathrm{~dB}$ higher on average than the $\mathrm{CB}$ thresholds at frequencies below $20 \mathrm{kHz}$, with larger differences in sensitivity at higher frequencies. These differences in threshold are all significant at the $5 \%$ level or better. There is no correlation be- 

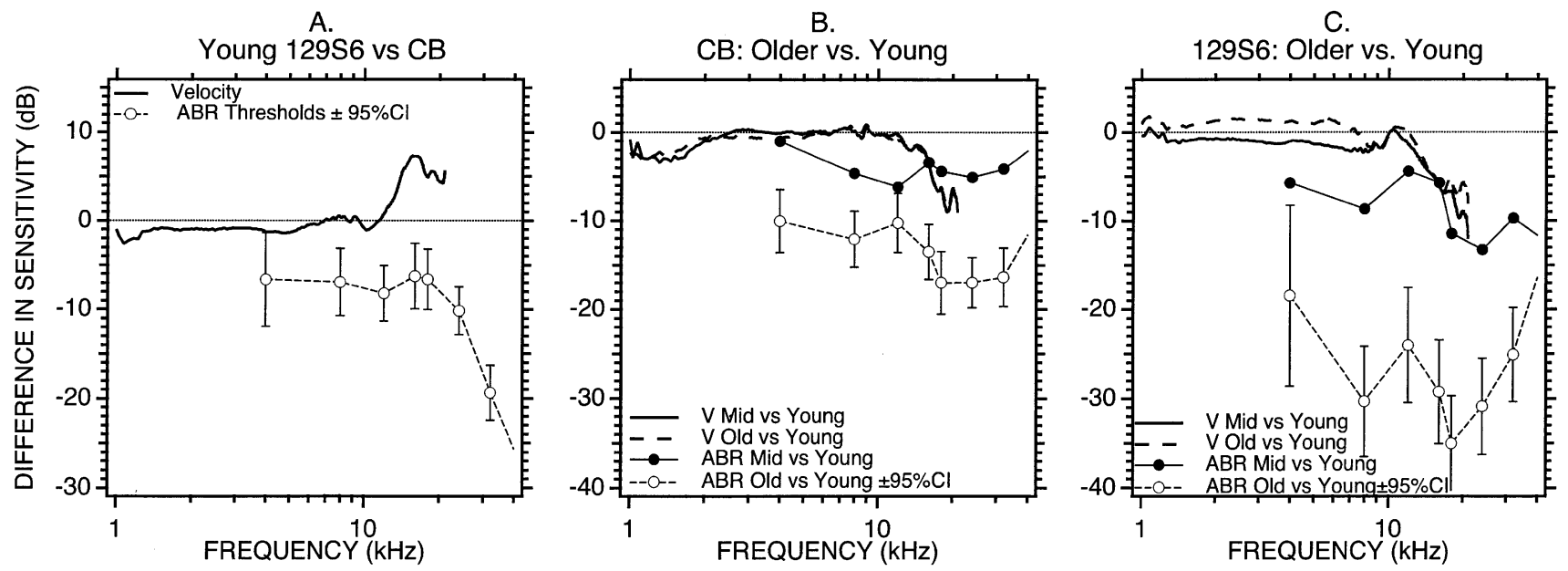

FIG. 6. Comparisons of strain- and age-related differences in ABR and middle-ear sensitivity. The curves are plotted as $\mathrm{dB}$ differences, where the $A B R$ data are differences in threshold between the base condition and the test condition, and the umbo velocity data are shown as $20 \log 10$ (velocity in the test condition/velocity in the base condition). The differences are defined so that a loss in sensitivity relative to the base condition plots below $0 \mathrm{~dB}$. The error bars around the ABR data are 95 confidence intervals calculated assuming that the variances in the base and test groups are equal. A. A comparison of the strain difference between young CB (the base condition) and 12956 (the test condition) mice in sensitivity of the middle ear (solid lines) and ABR (dashed lines and circles). The positive $\mathrm{dB}$ values between 10 and $20 \mathrm{kHz}$ are indicative of

tween the differences in the sensitivity of the middle ear and ABR measurements in these young ears from the two strains.

Similar comparisons of the changes in middle-ear and $A B R$ sensitivity with age in the two strains are shown in Figures 6B (CB) and 6C (129S6). The ABR data in both panels suggest a progressive increase in auditory thresholds (and decrease in sensitivity) with age in the two strains, with the 129S6 strain generally showing larger threshold increases with age. The changes in middle-ear sensitivity in both strains are restricted to frequencies above $10 \mathrm{kHz}$ and seem to occur primarily between the young- and middle-age group. The magnitudes of these age-related changes in middle-ear function are small (less than $10 \mathrm{~dB}$ ) compared to the 5-35 dB changes in sensitivity seen in the ABR measurements with age. These data suggest that differences in middle-ear and ABR sensitivity are not correlated between the two strains, nor is there a correlation between age-induced alterations in middle-ear function and ABR thresholds within the two strains.

\section{Umbo velocity in pathologic ears}

We also measured umbo velocity transfer functions in ears with various pathologies so that we could esti- increased motion of the umbo in 12956 young as compared to the CB. B. A comparison of the age-related differences in the three age groups of $C B$ mice, where the umbo velocities and $A B R$ measured in the young mice act as the base and the middle-age and old-age mice are the test conditions. The curves indicate a slight reduction in the umbo velocity at high frequencies in the two older groups and a larger increase in ABR thresholds with age. C. A comparison of the age-related differences in the three age groups of 12956 mice, where the umbo velocities and ABR measured in the young mice act as the base and the middle-age and old-age mice are the test conditions. The curves indicate a slight reduction in the umbo velocity at high frequencies in the two older groups and a much larger increase in ABR thresholds with age.

mate the effect of such changes on middle-ear function. Figure 7 is a comparison of velocity measurements from the mean middle-age group of NORMAL 129S6 and measurements in pathological individuals in the different groups. A cloudy TM with no middle-ear fluid or incomplete fluid filling had little impact on the measured umbo velocity; the velocity magnitudes in these conditions were generally a little lower than the mean normal velocities for their age and strain. The largest difference with a cloudy TM occurred in the case with partial fluid filling at frequencies less than $10 \mathrm{kHz}$ where the magnitude was $50-70 \%$ of normal. The edematous TM showed a small reduction in velocity at frequencies $<10 \mathrm{kHz}$ with a much larger reduction (as much as a factor of 100) at frequencies above $10 \mathrm{kHz}$. In contrast to the relatively small losses observed with a cloudy TM and an aerated middle ear, opaque and colored TMs with middle ears full of clear or purulent fluid were associated with large reductions (factors of 3-100) at all frequencies.

\section{Age- and strain-dependent variations in the incidence of middle-ear pathology}

We observed a strong relationship between the incidence of ear pathology and strain and age. Table 6 


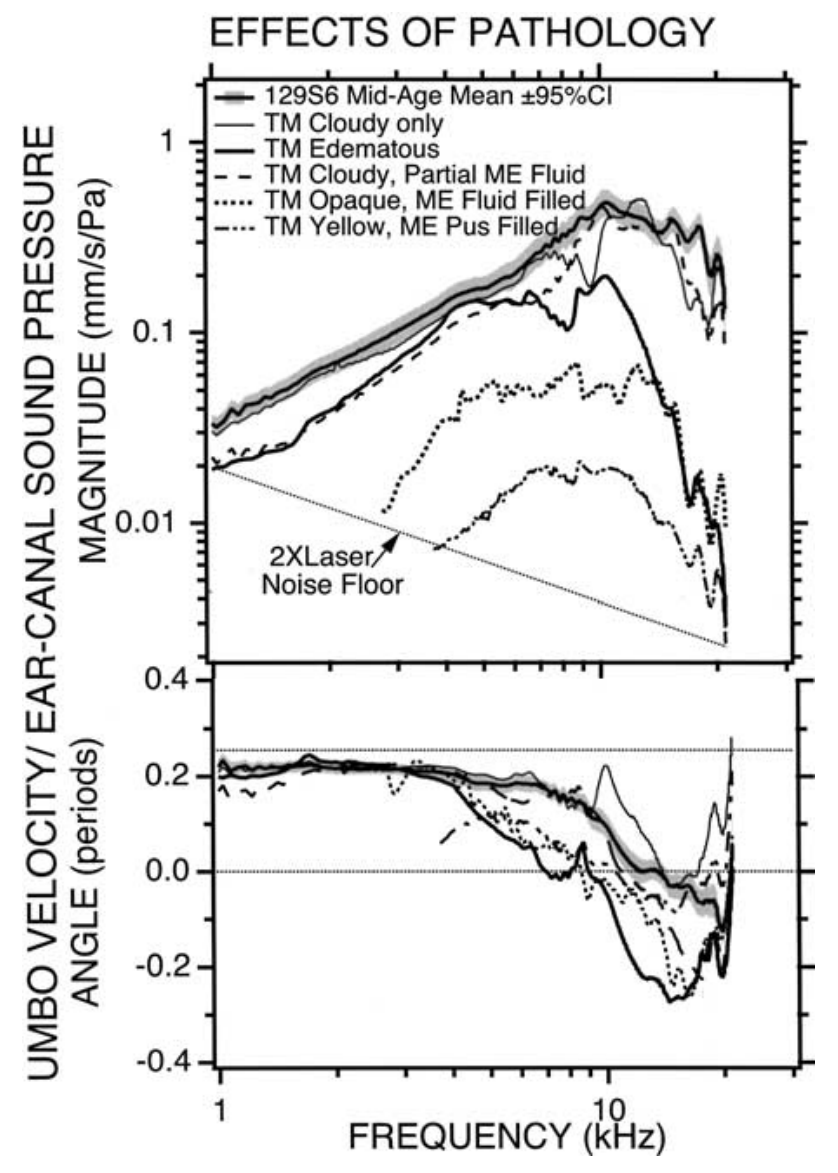

FIG. 7. Examples of the effect of pathology on the umbo velocity transfer function. Measurements in different pathological ears are compared to the mean of the middle-age 12956 group. The TM cloudy-only measurement was made in a young 129S6. The TM edematous measurement comes from an old CB. The TM opaque with middle-ear fluid measurement was made in a mid-aged 129 S6. The other measurements were made in old 12956 mice.

demonstrates that there was a larger percentage of pathological ears in the 129S6 strain than in the CB strain. Furthermore, the incidence of pathology in the 129S6 strain was largest in the oldest group. The significance of the observed variations in incidence of pathology was estimated using $\Gamma^{2}$ analyses. The analyses demonstrated that the incidence of pathology is unequal among the six groups of strain and age with a probability of equal incidence of $p<0.005$. These analyses also demonstrated a highly significant difference $(p<0.005)$ between the incidences of pathology in the two strains regardless of age, with the higher incidence in the 129S6 (25 of 63 ears as opposed to 4 of $33 \mathrm{CB}$ ears). There is little difference in the incidence of pathology between the young and the middle groups of the two strains, but there is a significant strain-associated difference in the incidence of pathology in the oldest groups $(p<0.005)$ with the higher susceptibility to disease in the old 129S6 (68\%, 15-22 ears) group compared with the old CB group (17\%, 2-12 ears).
Of note, $19 \mathrm{~F} 1$ offspring of $\mathrm{CB} \times 129 \mathrm{~S} 6$ crosses (all 18-24 months of age) were also evaluated for middleear involvement. All had normal-appearing TMs and middle ears and all had umbo velocities consistent with normal middle-ear function. We are currently following up on this observation of apparently recessive inheritance of the susceptibility to middle-ear pathology in 129S6.

\section{DISCUSSION}

Our discussion summarizes our results, compares them to similar studies in the literature, and discusses possible mechanisms for the age-related differences we found.

\section{The effect of age on middle-ear function in} normal ears

In general, the results summarized in Figures 3 and 5 are consistent with small but significant differences in sound-induced umbo velocity that are related to both strain and age. While we saw only small differences in umbo velocity at frequencies less than $10 \mathrm{kHz}$ as a function of either age or strain, at higher frequencies, the umbo velocity magnitude was significantly larger in the youngest groups (Table 5). The age-related difference was larger in the 129S6 strain, consistent with a factor of 2-3 $(6-10 \mathrm{~dB})$ difference in magnitude between 15 and $21 \mathrm{kHz}$. The age-related difference in magnitude at high frequencies was somewhat smaller in the CB.

A similar comparison of umbo velocity with age was performed in the BALB/c strain of mice by Doan et al. (1996a,b). Their results show a comparable increase in magnitude in the umbo velocity in ears of young mice as opposed to older mice (factors of 2-3 or 6-10 dB); however, the differences they report are most evident at lower frequencies $(f<15 \mathrm{kHz})$, whereas the differences we report are largest at frequencies between 12 and $21 \mathrm{kHz}$. Doan et al. (1996a) also report some age-related difference in velocity at frequencies greater than $21 \mathrm{kHz}$ (the highest frequencies of our measurements) but in general those differences were found to be insignificant. In summary, our and other data in the literature are consistent with a decrease in middle-ear function with increased age in mice of three strains. Those differences are, in general, smaller than 6-10 dB and appear to vary with frequency depending on the strain.

Interstrain differences in normal middle-ear function

Figures 3 and 5 suggest that the biggest difference in middle-ear function between the two strains we 
TABLE 6

measured was in the larger high-frequency umbo velocity in young 129S6 compared with the velocity observed in young CB. These data also suggest that umbo velocity is more similar in the older animals from the two strains. The significant high-frequency difference in the young animals is not consistent with an earlier report of similar umbo velocity in these two strains (Yoshida et al. 2000). Those measurements are much like the data in this study at frequencies below $12 \mathrm{kHz}$, but the earlier measurements show a fairly sharp peak in umbo velocity transfer function near 20 $\mathrm{kHz}$ that is not observed here. This difference may be due to differences in method. In the earlier study, sound pressure was measured just outside the bony ear canal with a microphone assembly integrated within the sound coupler. In the present study the sound pressure at the TM was measured with a movable probe tube microphone placed within the entrance of the bony ear canal. Control measurements made while moving the probe microphone around the coupler space are consistent with the existence of standing waves within the coupler at the location of the previous fixed microphone. These waves seem to have contaminated the previous sound pressure estimates, yielding a "zero" near $20 \mathrm{kHz}$ at the microphone that led to an underestimate of TM sound pressure at that frequency and an overestimate of the umbo velocity transfer function in the Yoshida et al. (2000) data. As for other mouse strains, comparisons between the present results and velocity measurements from different strains (BALB/c: Doan et al. 1994, 1996a,b; C57BL/6J: Saunders and Summers 1982) indicate a great deal of similarity in the middleear function among the different strains that have been tested.

\section{The effect of age on the ABR}

The ABR data in this report show clear age-related increases in threshold (Fig. 6) in both the CB and the 129S6 strains, where the changes in 129S6 thresholds with age are significantly (10-20 dB) larger. CBA-derived strains have been used as a standard for hearing in mice because their ears are less affected by aging (Zheng et al. 1999; Jimenez et al. 1999), though Li and Borg (1991) have noted broadband 20-40 dB threshold differences between young and 24-monthold CBA/ca (a different substrain from our CBA/ $\mathrm{CaJ})$. At younger ages, including 12 and 18 months, the CBA/ca mice of Li and Borg (1991) show a primarily high-frequency loss of as much as $20 \mathrm{~dB}$ relative to young animals. These changes in 12-24month-old $\mathrm{CBA} / \mathrm{ca}$ mice are larger than the ABR threshold increases observed in the middle- and oldage $\mathrm{CBA} / \mathrm{CaJ}$ in this report. On the other hand, the age-related changes we observe in ABRs are much larger than the minimal losses in distortion-product otoacoustic emissions (DPOAE) in CBA/CaJ of similar ages that have been reported by Jimenez et al. (1999). This difference between these ABR results in our CB group and other reports in the literature will be better addressed when our larger ongoing study investigating age-related changes in ABR and DPOAE is completed.

\section{Comparisons of differences in $\mathrm{ABR}$ and umbo velocity in the two strains and three age groups}

The mean strain-related differences in ABR threshold and umbo velocity measured in young CB and $129 \mathrm{~S} 6$ (Fig. 6A) are contradictory. The middle-ear measurements suggest a superior sensitivity of the 129S6 middle ear above $12 \mathrm{kHz}$, while the ABR measurements suggest a superior sensitivity of the CB auditory periphery at all frequencies, with the largest differences in threshold at the higher frequencies. The basis for this contradiction is unknown.

The age-related changes in ABR in the two populations are clearly significant, with the larger changes occurring in the older animals and in 129S6 vs. CBs of similar age (Figs. 6B,C). These large changes are in the same direction as the observed reductions in the magnitude of the umbo velocity transfer function at frequencies above $10 \mathrm{kHz}$ in the aged mice. However, the changes in ABR with age, particularly in the oldage group, are much larger than the age-related 
changes in middle-ear function we observe. We can only conclude that only a small part of the effect of age on ABR in these two strains can be attributed to aging of the middle ear.

\section{Interstrain and age-related differences in} incidence of middle-ear pathology

McGinn et al. (1992) have reported differences in the susceptibility to middle-ear disease between two CBA substrains $(\mathrm{CBA} / \mathrm{J}$ and $\mathrm{CBA} / \mathrm{CaJ})$, in which the incidence of middle-ear pathology was much greater in CBA/J. Our results (Table 6 and the $\Gamma^{2}$ analyses described above) are consistent with (1) a strain-related difference in the susceptibility of the middle ear to pathology, with the 129S6 strain being much more susceptible to pathology; (2) a low incidence of middle-ear pathology in our CB (CBA/CaJ) population, consistent with McGinn et al. (1992); and (3) an increased incidence of middle-ear disease in the older 129S6, where the middle ears of 10 of 41 (24\%) young and mid-age 129S6 showed pathology compared to 15 of $22(68 \%)$ from the old 129S6 group. The incidence of disease in the old 129S6 group is much higher than that in the old CB group (17\%). Humans also display an increased incidence of chronic middle-ear disease with age (Rudin et al. 1985).

We also observed a separation of the different pathological types within the age and strain groups. The mid- and old-age pathologic CB middle ears (all young CB mice were normal) generally were due to a cloudy or edematous TM with the middle ear free of fluid. The pathologic young 129S6 ears also had cloudy or edematous TMs with air-filled middle ears; however, all but one of the middle- and old-age 129S6 ears with pathology had fluid behind the TM and many had an opaque or yellow-colored TM. This large difference in the incidence of middle-ear disease in the two strains suggests that $\mathrm{CBA} / \mathrm{CaJ}$ is a superior strain for auditory research, especially in aging studies.

\section{The effect of pathology on middle-ear function}

The pathological changes we observed fit into two basic groups: (1) TM changes with no accumulation of middle-ear fluid, and (2) TM changes with accumulation of middle-ear fluid. While the clouding of the TM caused small decreases in umbo velocity at all frequencies, an edematous TM with no middle-ear fluid resulted in large decreases in the high-frequency response of the umbo. Partial fluid filling of the middle ear produced larger low-frequency changes. The combination of a cloudy TM and partial middleear fluid produced a factor of 2 decrease in the lowfrequency umbo velocity and little change in the high frequencies. Complete filling of the middle ear with clear or purulent fluid, coupled with an opaque or yellow-colored TM, was associated with broadband decreases of factors of 3-100 in umbo velocity magnitude (Fig. 7). The magnitude of these changes is similar to the hearing loss produced by otitis media in human patients (Fria et al. 1985).

Relationship between our data and the effect of age on middle-ear function in humans

As previously reviewed by Doan et al. (1996a), the existence of a change in middle-ear function with increasing age in humans has been a point of controversy. Morphologic studies have demonstrated changes in the incudomallear joints (Etholm and Belal 1974) and TMs (Ruah et al. 1991) of aging humans. In terms of functional measurements, Nixon et al. (1962) suggested the presence of an age-related conductive hearing loss in humans of more than $10 \mathrm{~dB}$ at $4 \mathrm{kHz}$ in a group of 55 year olds. However, tympanometric studies and multifrequency impedance measurements in young and aged humans generally do not indicate any age-related difference in middle-ear function (Thompson et al. 1979; Holte 1991; Wiley et al. 1999). Although some studies present evidence of a small decrease in middle-ear compliance with age (e.g., Gates et al. 1990), another presents evidence of an age-related increase in middle-ear compliance (Beattie and Leamy 1975). There is also a report of an age-related change in the damping within the tympanic membrane (Gaihede et al. 2000).

In the mouse we observe significant age-related changes in umbo velocity only at frequencies above 12$14 \mathrm{kHz}$ (Figs. 3 and 5, Table 4) and not at the lower frequencies that are dominated by middle-ear compliance. In general, tympanometric studies are most sensitive to changes in compliance and are probably not sensitive to the small middle- and high-frequency changes we observed in the two strains of mice. The best evidence of an age-related change in middle-ear function at frequencies above the middle-ear corner frequency in humans is the age-related conductive loss observed by Nixon et al. (1962). Their measured 10-dB air bone gap at $4 \mathrm{kHz}$ in humans is a reasonable match in magnitude to the age-related loss observed in our study at $15-20 \mathrm{kHz}$. Therefore, while there is evidence for and against an age-related change in middle-ear function in humans, some of the evidence for such a change has features in common with the middle-tohigh-frequency alterations observed in this study.

\section{SUMMARY}

Measurements of umbo velocity made on two different strains of mice, 129S6/SvEvTac and CBA/CaJ, 
within three different age ranges indicate small decreases in middle-ear sensitivity with increasing age. The decreases occur in the $12-20-\mathrm{kHz}$ frequency range and occur between 3 and 12 months of age. There is also a strain difference in sound-induced umbo velocity, with the young 129S6 having more sensitive middle-ear function than the $\mathrm{CB}$; this strain difference in middle-ear sensitivity decreased with increasing age. These results support the existence of a small age-related loss in middle-ear sensitivity in mouse first described in the BALB/c strain by Doan et al. (1996a,b). Neither the strain-related differences in middle-ear sensitivity nor the age-related decreases in middle-ear sensitivity correlate with measured difference in ABR thresholds in the three age groups of the two strains. A significant increase in the probability of middle-ear pathology was noted for the oldest age group of $129 \mathrm{~S} 6$ mice, where $68 \%$ of mice examined for this study had some signs of middle-ear pathology. This compares with an incidence of pathology of $0-20 \%$ in CBs of the three age groups. No relationship between the incidence of pathology and age was observed in the CB strain.

\section{ACKNOWLEDGMENTS}

This work was supported by grants from the National Institutes of Health to JJR, SGK, and BLT. We thank L. Robinson of the Virginia Merrill Bloedel Hearing Research Center, University of Washington, for excellent care in maintaining the mouse colonies. We also thank the core engineering staff of the Eaton-Peabody Laboratory, Massachusetts Eye and Ear Infirmary, for their technical support. We are grateful to M.C. Liberman (Eaton-Peabody Lab, Massachusetts Eye and Ear Infirmary) and two anonymous reviewers for comments on an earlier version of this manuscript.

\section{REFERENCES}

BeAtTiE R, LeAmy D. Otoadmittance: Normative values, procedural variables and reliability. J. Am. Audiol. Soc. 1:21-27, 1975.

DoAn DE, Cohen YE, SAunders JC. Middle-ear development: IV. Umbo motion in neonatal mice. J. Comp. Physiol. A 174:103110, 1994.

Doan DE, Erulkar JS, Saunders JC. Functional changes in the aging mouse middle ear. Hear. Res. 97:174-177, 1996a.

Doan DE, IgIC PG, SAunders JC. Middle-ear development VII: Umbo velocity in the neonatal rat. J. Acoust. Soc. Am. 99:15661572, 1996b.

Etholm B, Belal Jr A. Senile changes in the middle ear joints. Ann. Otol. 83:49-54, 1974.

Fria TJ, CANTEkIN EI, Eichler JA. Hearing acuity of children with otitis media with effusion. Arch. Otolaryngol. 111:10-16, 1985.

Gaihede M, Koefoed-Nielsen B. Mechanics of the middle ear system: age-related changes in viscoelastic properties. Audiol. Neurootol. 5:53-58, 2000.
Gates GA, Cooper Jr JC, Kannel WB, Miller NJ. Hearing in the elderly: the Framingham cohort, 1983-1985. Part I. Basic audiometric test results. Ear Hear. 11:247-256, 1990.

Holte L, Margolis RH, Cavanaugh JR RM. Developmental changes in multifrequency tympanograms. Audiology 30:1-24, 1991.

Huangfu N, Saunders JC. Auditory development in the mouse: structural maturation of the middle ear. J. Morphol. 176:249259, 1983.

Jimenez AM, Stagner BB, Martin GK, Lonsbury-Martin BL. Agerelated loss of distortion product otoacoustic emissions in four mouse strains. Hear. Res. 138:91-105, 1999.

Kujawa SB, Tempel BL, Golden AS, Liberman MC. Noise resistance and AHL in the 129S6/SvEvTac mouse are inherited recessively. Assoc. Res. Otolaryngol. Abs. 28, 2001.

Kujawa SG, Street VA, Liberman MC, Tempel BL. Characterizing the genetics of noise resistance in 129S6/SvEvTac mice. Assoc. Otolaryngol. Abs. 2, 2002.

Li H-S, Borg E. Age-related loss of auditory sensitivity in two mouse genotypes. Acta Otolaryngol. 111:827-834, 1991.

Li H-S, Borg E. Auditory degeneration after acoustic trauma in two genotypes of mice. Hear. Res. 68:19-37, 1993.

Lynch III TJ, Peake WT, Rosowski JJ. Measurements of the acoustic input impedance of cat ears: $10 \mathrm{~Hz}$ to $20 \mathrm{kHz}$. J. Acoust. Soc. Am. 96:2184-2209, 1994.

MCGinn MD, BEAN-KNudsen D, ERmel RW. Incidence of otitis media in CBA/J and CBA/CaJ mice. Hear. Res. 59:1-6, 1992.

Nixon JC, Glorig A, High WC. Changes in air and bone conduction thresholds as a function of age. J. Laryngol. Otol. 76:288298, 1962.

Parkinson NJ, Olsson CL, Hallows JL, McKee-Johnson J, Keogh K, Noben-Trauth K, Kujawa SG, Tempel BL. Mutant $\beta$-spectrin 4 causes auditory and motor neuropathies in quivering mice. Nat. Genet. 29:61-65, 2001.

Ruah CB, Schachern PA, Zelterman D, Paparella MM, Yoon TH. Age-related morphologic changes in the human tympanic membrane. Arch. Otolaryngol. Head Neck Surg. 117:627-634, 1991.

Relkin EM, SAunders JC. Displacement of the malleus in neonatal golden hamsters. Acta Otolaryngol. 90:6-15, 1980.

Rudin R, Welin L, SväRdsudd K, Tibblin G. Middle ear disease in samples from the general population: II. History of otitis media and otorrhea in relation to tympanic membrane pathology; The study of men born in 1913 and 1923. Acta Otolaryngol. 99:53-59, 1985.

SAunders JC, Summers RM. Auditory structure and function in the mouse middle ear: An evaluation by SEM and capacitive probe. J. Comp. Physiol. A 146:517-525, 1982.

Thompson DJ, Sills JA, Recke KS, Bui DM. Acoustic admittance and the aging ear. J. Speech Hear. Res. 22:29-36, 1979.

Wiley Tl, Cruickshanks KJ, Nondahl DM, Tweed TS. Aging and middle ear resonance. J. Am. Acad. Audiol. 10:173-179, 1999.

Willott JF, Seegers Bross L, McFadden SL. Morphology of the dorsal cochlea nucleus in C57BL/6J and CBA/J mice across the life span. J. Comp. Neurol. 321:666-678, 1992.

Willott JF, Turner JG, Sundin VS. Effects of exposure to an augmented acoustic environment on auditory function in mice: roles of hearing loss and age during treatment. Hear. Res. 142:79-88, 2000.

Yoshid A, Hequembourg SJ, Atencio CA, Rosowski JJ, Liberman MC. Acoustic injury in mice: $129 / \mathrm{SvEv}$ is exceptionally resistant to noise-induced hearing loss. Hear. Res. 141:97-106, 2000.

Zheng QY, Johnson KR, ERWAY LC. Assessment of hearing in 80 inbred strains of mice by ABR threshold analysis. Hear Res. 130:94-107, 1999. 